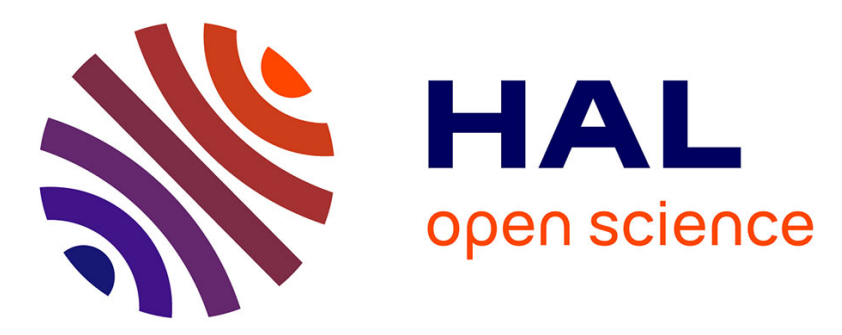

\title{
Hydrogen - Plasticity interactions : modelling and experiments in hydrogenated nickel alloys
}

\author{
David Delafosse, Gouenou Girardin
}

\section{To cite this version:}

David Delafosse, Gouenou Girardin. Hydrogen - Plasticity interactions : modelling and experiments in hydrogenated nickel alloys. 5th Euromech Solid Mechanics Conference, Aug 2003, Thessalonique, Greece. pp.151, 10.1515/JMBM.2005.16.3.151 . emse-01074021

\section{HAL Id: emse-01074021 \\ https://hal-emse.ccsd.cnrs.fr/emse-01074021}

Submitted on 12 Oct 2014

HAL is a multi-disciplinary open access archive for the deposit and dissemination of scientific research documents, whether they are published or not. The documents may come from teaching and research institutions in France or abroad, or from public or private research centers.
L'archive ouverte pluridisciplinaire HAL, est destinée au dépôt et à la diffusion de documents scientifiques de niveau recherche, publiés ou non, émanant des établissements d'enseignement et de recherche français ou étrangers, des laboratoires publics ou privés. 


\title{
HYDROGEN - PLASTICITY INTERACTIONS: MODELLING AND \\ EXPERIMENTS IN HYDROGENATED NICKEL ALLOYS
}

\section{David DELAFOSSE, Gouenou GIRARDIN}

Centre SMS, UMR CNRS 5146, Ecole Nationale Supérieure des Mines, 158 Cours Fauriel, 42023 Saint-Etienne cedex 02, France

david.delafosse@emse.fr

\begin{abstract}
The effect of hydrogen on tensile tests of nickel and binary nickel - 16 wt. \% chromium is analysed in terms of solute drag phenomenon. Static Strain Ageing experiments are used to measure the saturated dislocation pinning force as a function of the $\mathrm{H}$ concentration. First order hydrogen - dislocation interactions causes a shielding of the pair interactions between edge dislocations. The influence of this screening effect is analytically evaluated on the selfenergy and line tension of curved dislocations, the critical force for the expansion of a dislocation loop and the dissociation mechanism. These results are used to interpret experimental results on the plastic flow of hydrogen-charged nickel single crystals oriented for easy glide. This study illustrates the mechanisms of $\mathrm{H}$ - dislocation interactions and their consequences on the different contributions of hydrogen to the flow stress of nickel.
\end{abstract}

Keywords: ageing, dislocations, hydrogen, plasticity, modelling 


\section{Introduction}

In austenitic alloys, hydrogen assisted fracture at low temperature and various instances of Stress Corrosion Cracking (SCC) stem from the synergistic interactions between absorbed hydrogen and crack-tip plasticity. The first level of interaction is the trapping and transport of solute hydrogen in the hydrostatic stress field of mobile dislocations. Tritium desorption measurements during straining and autoradiographic observations on strained samples provide the most direct experimental evidence of such transport mechanims [1, 2]. A reverse mechanism of coupling arises from the dilatation strains induced by solute hydrogen in the host lattice, and the distribution of solutes in the hydrostatic stress gradient surrounding edge dislocations. This results in the screening of the pair interactions beween neighbouring dislocations in the presence of solute hydrogen. Hydrogen is thus expected to affect crack-tip plasticity. The Hydrogen Enhanced Localized Plasticity (HELP) mechanism for hydrogen assisted fracture [3] and the Corrosion Enhanced Plasticity Model (CEPM) for the Stress Corrosion Cracking of austenitic stainles steels in chloride containing environments [4] specifically account for the strain localization that would be expected from such hydrogen effects at a crack tip.

The screening of pair interactions by diffusing hydrogen is now well modelled in linear elasticity. Starting from Larché and Cahn's formalism, Sofronis and Birnbaum [5, 6] have given a thorough analytical and numerical treatment of the coupled elasticity - diffusion problem for solute hydrogen in metals, which also accounts for the coupling to the second order that results from the alteration of elastic constants in the presence of solute atoms. Focussing only on the first order level of coupling, which stems from the dilatation strains induced by solute hydrogen in the host lattice, we have developed an alternative simulation scheme [7] based on a discretisation of the hydrogen distribution into an array of discrete line 
defects. Introducing a "screening index" $\mathrm{S}$ that measures the relative decrease of the pair interactions in the presence of hydrogen, it was shown that, to the first order, $\mathrm{S}$ is a function of the temperature $\mathrm{T}$, the hydrogen atomic concentration $c=n_{H} / n_{M}$ and material parameters contained in the $\beta$ coefficient (Eq. 1). A general expression for the relative screening of the resolved shear stress between the edge components of two dislocations is given by:

$$
S(T, c)=\frac{S_{0}}{1+\beta \frac{T}{c}} \text { with } S_{0}=75 \%, \text { with } \beta=2.3310^{-4} \mathrm{~K}^{-1} \text { for nickel. }
$$

In situ transmission electron microscopy in an environmental cell under hydrogen partial pressure has demonstrated the effect of hydrogen on the mobility of isolated dislocations, on the dislocation density in pile-ups under stress, as well as other plasticity mechanisms such as the cross-slip of screw dislocations [8]. However, the situation appears more confused when addressing the issue of the role of hydrogen on the macroscopic flow stress, especially in $f c c$ materials. While most experimental studies of of the plastic flow in hydroganated FCC metals agree on an increased localisation of plasticity associated to solute hydrogen (such as in, e.g.: [9]), measurements of the effects of hydrogen on the macroscopic flow stress yield contradictory results, with a majority of studies pointing out a net hardening effect. In these alloys, the macroscopic flow stress is governed by the reactions between dislocations rather than their long range elastic interactions. Tensile test data must therefore be interpreted in terms of this collective behaviour. In this paper, we present analytical and experimental results that illustrate the possible effects of hydrogen in solid solution on plasticity mechanisms, and their consequences on the tensile flow stress of H-containing nickel.

\section{Experimental measurement of the solute drag effect}


A direct manifestation of solute - dislocations interactions in many dilute alloys is given by the Portevin - Le Chatelier (PLC) effect, i.e.: the occurrence of jerky flow within a bounded range of temperature and plastic strain rate. In hydrogen-containing nickel, it was studied by Boniszewski and Smith [10], Combette and Grilhé [11] and Wilcox and Smith $[12,13]$. The PLC effect is related to the dynamic interaction between diffusing solute atoms and mobile dislocations, i.e. to the Dynamic Strain Ageing (DSA) phenomenon. Increasing the strain rate causes a decrease of the waiting time of dislocations, thus reducing the amount of solute ageing, when the apparent mobility of dislocations and that of solutes are of the same order of magnitude. The net effect is a negative contribution to the total Strain Rate Sensitivity (SRS) of the flow stress. The onset of plastic instabilities (jerky flow) occurs when the SRS turns negative and the PLC effect is thus observed within a subdomain of the DSA domain.

In order to study the solute drag phenomenon, we performed static strain ageing (SSA) experiments. SSA refers to the transient stress peak observed in hydrogenated nickel when a prestrained specimen is unloaded and aged for a prescribed time and then reloaded at the initial strain rate (figure 1). This effect is related to the pinning of dislocations by diffusing atoms during the ageing time. The main difference between static and dynamic strain ageing lies in the fact that in the former case the ageing time is prescribed, while in the later the waiting time is indirectly prescribed by the plastic strain rate. Furthermore, such SSA measurements can only be performed at a temperature below that of the PLC effect for the strain rate of interest, i.e.: when diffusing solutes can no longer cope with mobile dislocations during continuous straining.

Cylindrical tensile specimens with a $2.5 \mathrm{~mm}$ radius and a $25 \mathrm{~mm}$ gauge length were cut in pure nickel and a binary nickel 16 wt. \% chromium containing less than 15 wt. ppm C, less 
than 10 wt. ppm O, N and less than 5 wt. ppm S. Samples were hydrogenated thermally in an autoclave in order to get two hydrogen levels (referred to as "high" and "low" in the following). The "high" level of hydrogen charging of samples was achieved by a 48 hours exposure to a 150 bar pressure of $\mathrm{H}_{2}$ gas at $450^{\circ} \mathrm{C}$ in an autoclave and resulted in a 1900 at. ppm concentration both in pure nickel and in Ni-16Cr. The "low" level corresponded 100 bar pressure of $\mathrm{H}_{2}$ gas at $350^{\circ} \mathrm{C}$, giving a 1500 at. ppm in both metals. After charging, the specimens were air-cooled during $2 \mathrm{mn}$, water-quenched, and further cooled in liquid nitrogen. They were stored in a liquid $\mathrm{N}_{2}$ tank before mounting and mechanical testing. Tensile test was performed in a strain rate and temperature domain inside that of the DSA, on the "low temperature" side of the PLC subdomain. For an initial strain rate of $10^{-2} \mathrm{~s}^{-1}$, the corresponding temperature was $-140^{\circ} \mathrm{C}$ for nickel and $-100^{\circ} \mathrm{C}$ for $\mathrm{Ni}-16 \mathrm{Cr}$. After prestraining up to a strain of $7 \%$, the specimen was unloaded down to $5 \mathrm{MPa}$ and aged during a hold time $t_{h}$.

Figure 2 describes the evolution of the ageing peak stress $\Delta \sigma$ with respect to the ageing time for nickel and the binary $\mathrm{Ni}-16 \mathrm{Cr}$ alloy. These curves exhibit a behaviour consistent with the modelling of static strain ageing by the Kubin and Estrin [14] "dislocation model" and with the recent work of De et al. on ultra low carbon bake-hardening steel [15]. For Ni-16Cr, figure 2.a shows that $\Delta \sigma$ increases and eventually saturates with increasing ageing times. $f_{0}$, the saturated value of $\Delta \sigma$, is almost reached at the same ageing time for both concentrations, but the magnitude of $f_{0}$ is an increasing function of it. For the 1900 at. ppm $\mathrm{H}$ content, the saturation is reached after $10^{4} \mathrm{~s}$ and $f_{0}$ is about $16 \mathrm{MPa}$ whereas it is about $13 \mathrm{MPa}$ for a concentration of 1500 at. ppm. Fig. 2.b illustrates the evolution $\Delta \sigma$ for nickel. The magnitude of $\Delta \sigma$ presents the same behaviour as for the alloy, but the saturation is not reached for an ageing time of 8 hours at $-140^{\circ} \mathrm{C}$. We expect the saturation to be reached after $10^{5} \mathrm{~s}$ and to be slightly higher than for the alloy. 
Since $f_{0}$ is the saturated value of $\Delta \sigma$ that can be produced by the ageing mechanism, it measures the maximum pining stress solute hydrogen and mobile dislocations during straining. At high (room) temperature, where mobile dislocations are continuously aged during straining, the "solute drag" force corresponds to a constant hardening contribution to the flow stress of the same order of magnitude (15 to $20 \mathrm{MPa}$ ).

\section{Modelling of hydrogen effects on plasticity mechanisms}

In the following section, we evaluate the influence of solute hydrogen on the properties of individual dislocations, by considering $\mathrm{H}$ effects on the orientation dependence of the line energy. Before studying the influence of the screening index on dislocation loops and on dissociation, the line energy and the line tension is first examined.

Considering an initially straight dislocation with a "bow-out" along its line, we evaluated the energy increase caused by this incremental bend. Following the classical model of de Wit and Koehler [16], the self energy per unit length of a dislocation line depends on the orientation through the angle $\theta$ between the Burgers vector and the original straight line. Integrating the elastic interactions between incremental segments along a dislocation line, its energy per unit length is given by:

$$
E(\theta)=\frac{\mu b^{2}}{4 \pi K(\theta)} \ln \left(\frac{R}{r_{0}}\right) \text {, with } \frac{1}{K(\theta)}=\cos ^{2} \theta+\frac{\sin ^{2} \theta}{1-v}
$$

where $\mathrm{b}$ is the Burgers vector magnitude, $\mu$ the shear modulus, $v$ the Poisson ratio, $\mathrm{R}$ and $\mathrm{r}_{0}$ are, respectively, the outer and inner cut-off radii. Introducing the screening index $\mathrm{S}(T, c)$ 
defined by the expression (1) to account for hydrogen effects on the edge components, in the presence of hydrogen, the $\mathrm{K}(\theta)$ factor becomes:

$$
\begin{gathered}
\frac{1}{K_{\vartheta}(\theta)}=\cos ^{2} \theta+\frac{(1-\vartheta(T, c)) \sin ^{2} \theta}{1-v} \\
\text { with } \vartheta(T, c)=\frac{S(T, c)\left[1+3 v-2 v S(T, c)-4 S(T, c)+2 S(T, c)^{2}\right]}{1+v-2 S(T, c)}
\end{gathered}
$$

In the presence of hydrogen, the self-energy $\mathrm{E}(\theta)$ is as a function of the dislocation character and the screening index $\mathrm{S}(T, c)$. The variation of the self-energy per unit length is plotted on fig. 3.a for different values of S, for three different levels of shielding: $11 \%, 22 \%$ and $33 \%$ respectively, corresponding to $\mathrm{H}$ concentrations of $0.012,0.029$ and 0.055 respectively in nickel at room temperature. Predictably, by relaxing the hydrostatic component of the dislocations' stress tensor, hydrogen lowers the line energy of edge dislocations, while screw segments remain unaffected to the first order by hydrogen dilatation strains.

After de Wit and Koehler, the line tension is defined by the first derivative of the selfenergy with respect to the incremental increase in length caused by a small bow-out. The screening effect due to hydrogen gives the following expression for the line tension:

$$
\zeta_{\vartheta}(\theta)=\frac{\mu b^{2}}{4 \pi(1-v)}\left[1-\vartheta(T, c)-2(v-\vartheta(T, c))+3(v-\vartheta(T, c)) \cos ^{2} \theta\right] \ln \left(\frac{R}{r_{0}}\right)
$$

Fig. 3.b shows the line tension behaviour for different level of $S(T, c)$. The line tension of screw segments decreases with the increase of the screening index, as edge segments become more rigid. Consequently, a dislocation loop, which is elongated in the screw direction in an isotropic crystal without hydrogen tends toward isotropy as the screening effect increases. 
Based on the previous calculation, we evaluated the critical stress for the expansion of a dislocation loop. According to Hirth and Lothe [17], the shape of a circular dislocation loop was approximated by a hexagonal loop. In the presence of hydrogen, the expression for the total energy of a hexagonal loop of side L reads:

$$
W^{H}=\frac{3 \mu b^{2} L}{4 \pi(1-v)}\left[0.16(2-v-\boldsymbol{S}(\boldsymbol{T}, \boldsymbol{c}))+(2-v-\vartheta(\boldsymbol{T}, \boldsymbol{c})) \ln \left(\frac{L}{r}\right)\right]
$$

The force for the isotropic expansion of such a loop is the first derivative of the energy W with respect to the loop radius L. Expressing the ratio of this force in the presence of hydrogen $\mathrm{F}_{\mathrm{S}}$ to that of the force without hydrogen $\mathrm{F}$ gives the following simple expression as a function of $\mathrm{S}(T, c)$ :

$$
\frac{F^{H}}{F} \approx 1-0.58 S(T, c)
$$

Finally, we studied the influence of solute hydrogen on the dissociation of a perfect dislocation into Shockley partials. The Stacking Fault Energy (SFE), noted $\Gamma$, is a key parameter controlling the plasticity of $f c c$ alloys. In particular, the cross slip mechanism is governed by the SFE, a decrease of the SFE inducing a reduction of the cross-slip probability. It is observed experimentally that hydrogen promotes planar glide and increases the localisation of plastic strain [9]. The reason generally invoked is a decrease of the SFE induced by hydrogen. However, hydrogen also decreases the interactions between the edge components of mixed dislocations and these elastic interactions between solute atoms and dislocations are neglected in such analysis. The effects of the shielding of pair interactions between partial dislocations on the cross-slip mechanism are evaluated against possible $\mathrm{H}$ 
effects on the SFE.

In $f c c$ crystals, perfect dislocation of the type $1 / 2<110>$ dissociate into an extended dislocation consisting of two Shockley partials of the type $1 / 6<112>$, enclosing a stacking fault ribbon. Considering a perfect mixed dislocation, where the angle $\theta$ defines the character of the dislocation, partial dislocations are inclined at $\theta \pm \pi / 6$ to their respective lines. In the absence of external stress, the partials are in equilibrium at a distance $\mathrm{d}_{0}$ where each partial is submitted to two forces: the repulsion due to the other partial $(\alpha 1 / \mathrm{r})$ and the attraction due to the SFE. In the presence of hydrogen, the expression for the equilibrium separation between partials $\left(\mathrm{d}^{\mathrm{H}}\right.$ instead of $\mathrm{d}_{0}$ without hydrogen) is given by:

$$
d^{H}=\frac{\mu b_{p}^{2}}{8 \pi \Gamma} \frac{2(1-S(T, c))-(v-S(T, c))(1+2 \cos 2 \theta)}{1-v}
$$

where $b_{p}$ is the Burgers vector of the partials and $\theta$ the character of the perfect dislocation.

Assuming a constant value of the SFE, Fig. 4 depicts the evolution of the equilibrium ribbon width (in reduced units) as a function of the character of the initial perfect dislocation and the screening index. Focusing on the dissociation of a screw dislocation, we studied the cross-slip mechanism. Since the edge components of the Burgers vector of the partials have opposite Burgers vectors, they attract each other. Thus, by screening the attractive contribution, hydrogen induces a widening of the equilibrium dissociation distance. For a fixed value of the SFE and $\mathrm{S}=33 \%$, hydrogen induces a $28 \%$ increase of the ribbon width. Therefore, "elastic effects" contribute to the decrease of the cross-slip ability with increasing hydrogen contents. Ferreira provides one of the very few experimental measurements of hydrogen effects on the dissociation of perfect dislocations [18]. An analysis of these results that includes elastic screening effects is currently being conducted. 


\section{Experimental study of hydrogen effects on the plastic flow of nickel single crystals oriented for easy glide}

In this section, we present experimental results in tension of non-charged and hydrogencharged nickel single crystals oriented for single slip, which illustrate the above results. Smooth specimens with a 4-mm wide square section and a 10-mm gauge length were cut in a pure Ni single crystal along the [153] direction, corresponding to a "single slip" orientation. Samples were hydrogenated and stored in the conditions described above, giving a 1600 at. ppm hydrogen concentration. Stress strain curves for nickel and nickel-hydrogen at the initial strain rate of $10^{-2} \mathrm{~s}^{-1}$ are shown in fig. 5. Comparing the stress-strain curves of $\mathrm{Ni}$ and $\mathrm{Ni}-\mathrm{H}$ crystals, one can identify the following features related to hydrogen charging, along with our present interpretation of such features:

- Hydrogen causes a noticeable increase of the magnitude of $\tau_{\mathrm{I}}\left(\sim 0.0510^{-3} \mu\right)$. At room temperature, mobile dislocations are continuously aged during straining. This hardening effect induced by hydrogen is attributed to the viscous drag of Cottrell atmospheres.

- Hydrogen delays the emergence of stage II. In stage I ("easy glide"), a unique slip system is active. Plastic deformation during this stage is accommodated by the activation of FrankRead sources. Few obstacles impede the glide of emitted dislocation loops resulting in a very low work hardening rate. According to the results presented in the previous section, both multiplication and planar glide are expected to be favoured by solute hydrogen.

- In stage II, the introduction of hydrogen in nickel results in a significant increase of the work hardening. This stage of rapid linear hardening is characterized by a significant increase of the work hardening corresponding to the activation of secondary slip systems. The work hardening in stage II is governed by an interaction mechanism between 
dislocations from the first activated system and dislocations from secondary slip systems.

Delaying the emergence of stage II, hydrogen increase of the dislocation density stored during stage I. Thus causing an latent hardening of secondary slip systems.

These results provide experimental evidences of hydrogen effects on elementary plasticity mechanisms. The observed hydrogen effects on stage I (increase of $\tau_{\mathrm{I}}$ due to solute drag and extension of the planar glide) are consistent with recent works of Yagodzinskyy et al. [19] on 18Cr-16Ni-10Mn austenitic stainless steel single crystals with a much higher $\mathrm{H}$ content.

\section{Summary and conclusion}

In the first section, we studied the effect of solute drag on the flow stress of hydrogencontaining nickel and Ni-Cr. SSA experiments provided quantitative data to evaluate this phenomenon. The maximum pinning stress increases with the hydrogen concentration in pure nickel and Ni-16Cr. At room temperature, the corresponding solute drag yields a hardening contribution to the flow stress, up to $20 \mathrm{MPa}$ for a concentration of 1900 at. ppm.

Incorporating the shielding of pair interactions into classical results of the dislocation theory, we proposed analytical expressions for the effect of solute hydrogen on the following mechanisms and dislocation properties: the line energy and line tension of curved dislocations, the critical shear stress for the expansion of a dislocation loop and the cross-slip of screw dislocation. From these analytical results, we are currently developing numerical simulations to evaluate the role of hydrogen on the activity of a Frank-Read source, and on the stability of dislocation junctions. The purposes of such a detailed modelling are to provide quantitative tools for interpreting the mechanical testing of hydrogen containing samples in terms of dislocation mechanisms.

In the last part, we presented the experimental illustration of the mechanisms of $\mathrm{H}-$ 
dislocation interactions and their consequences on the different contributions of hydrogen to the flow stress of nickel single crystals oriented for easy glide. The following contributions were highlighted: (i) a positive contribution to the critical shear stress, due to the solute drag, (ii) an extension of the stage of easy glide that may be associated to a decrease of the crossslip probability, and (iii) a distinct increase of the work hardening rate in stage II in the presence of hydrogen, due to the latent hardening of secondary slip systems by extended primary glide.

More generally, the aim of this study is to validate the relevance of our modelling and simulation approach to the problem of the Stress Corrosion Cracking (SCC) of $f c c$ metals in acidic solutions, and possibly to suggest improvements of our simulation method which only considers "elastic effects" so far. This is expected to yield better predictive capabilities to our modelling efforts of the SCC of various engineering systems.

\section{References}

1. Brass, A.M. and J. Chene, Influence of deformation on the hydrogen behavior in iron and nickel base alloys : a review of experimental data. Materials Science and Engineering A, 1998. 242: p. 210-221.

2. Chene, J. and A.M. Brass, Hydrogen transport by mobile dislocations in nickel base superalloy single crystals. Scripta Materialia, 1999. 40(5): p. 537-542.

3. Birnbaum, H.K. and P. Sofronis, Hydrogen enhanced localized plasticity - a mechanism for hydrogen related fracture. Materials Science and Engineering A, 1994. 176: p. 191-202.

4. Magnin, T., A. Chambreuil, and B. Bayle, The corrosion-enhanced plasticity model 
for stress corrosion cracking in ductile fcc alloys. Acta Materialia, 1996. 44(4): p. 1457-1470.

5. Sofronis, P., The influence of mobility of dissolved hydrogen on the elastic response of a metal. Journal of the Mechanics and Physics of Solids, 1995. 43(9): p. 1385-1407.

6. Sofronis, P. and H.K. Birnbaum, Mechanics of the hydrogen-dislocation-impurity interactions--I. Increasing shear modulus. Journal of the Mechanics and Physics of Solids, 1995. 43(1): p. 49-90.

7. Chateau, J.P., D. Delafosse, and T. Magnin, Numerical simulations of hydrogen dislocations interactions in fcc stainless steels - Part I : hydrogen - dislocations interactions in bulk crystals. Acta Materialia, 2002. 50(6): p. 1507-1522.

8. Robertson, I.M., The effect of hydrogen on dislocation dynamics. Engineering Fracture Mechanics, 1999. 64: p. 649-673.

9. Abraham, D.P. and C.J. Altstetter, Hydrogen enhanced localization of plasticity in an austenitic stainless steel. Metallurgical and Materials Transactions A, 1995. 26A: p. $2859-2878$.

10. Boniszewski, T. and G.C. Smith, the influence of hydrogen on the plastic deformation, ductility and fracture of nickel in tension. Acta Metallurgica, 1963. 11: p. 165-178.

11. Combette, P. and J. Grilhé, Effet Portevin - Le Chatelier et discontinuité à la limite élastique dans le nickel hydrogéné. Mémoires Scientifiques de la Revue de Métallurgie, 1970. LXVII(7-8): p. 491-505.

12. Wilcox, B.A. and G.C. Smith, Acta Metallurgica, 1964. 12: p. 371.

13. Wilcox, B.A. and G.C. Smith, Intercrystalline fracture in hydrogen-charged nickel. Acta Metallurgica, 1965. 13: p. 331-343.

14. Kubin, L.P., Y. Estrin, and C. Perrier, On static strain ageing. Acta Metallurgica et 
Materialia, 1992. 40(5): p. 1037-1044.

15. De, A.K., S. Vandeputte, and B.C. De Cooman, Static strain aging behavior of ultra low carbon bake hardening steel. Scripta Materialia, 1999. 41(8): p. 831-837.

16. De Wit, G. and J.S. Koehler, Interaction of dislocations with an applied stress in anisotropic crystals. Physical Review, 1959. 116(5): p. 1113-1120.

17. Hirth, J.P. and J. Lothe, Theory of dislocations. Reprint ed. 1992, Malabar, Florida: Krieger Publishing Co.

18. Ferreira, P.J., I.M. Robertson, and H.K. Birnbaum, Influence of hydrogen on the stacking fault energy of an austenitic stainless steel, in Intergranular and interphase boundaries in materials. 1996, Transtech Publications: Switzerland. p. 93-96.

19. Yagodzinskyy, Y., O. Tarasenko, and H. Hanninen. Effect of hydrogen on plastic deformation of stable 18Cr-16Ni-10Mn austenitic stainless steel single crystals. in Hydrogen effects on materials behaviour and Corrosion Deformation Interactions. 2002. Moran, Wyoming: TMS, Warrendale, PA. 


\section{Figure captions}

Fig. 1: Static Strain Ageing experiments. Schematic representation of the transient peak stress $\Delta \sigma$ measured after a prescribed holding time $t_{\mathrm{h}}$ at near-zero applied stress.

Fig. 2: Evolution of the ageing peak stress $\Delta \sigma$ at $7 \%$ prestrain as a function of the ageing time for a) $\mathrm{Ni}-16 \mathrm{Cr}$ at $-100^{\circ} \mathrm{C}$ and b) pure nickel at $-140^{\circ} \mathrm{C}$ with the hydrogen concentration.

Fig. 3: Evolution of a) the self-energy per unit length and b) the line tension of a dislocation, both normalised by $\mu b^{2}$, as a function of the dislocation character with the screening index $\mathrm{S}(T, C)$.

Fig. 4: Evolution of the equilibrium dissociation distance with the screening index, normalised by $\mu b_{p}{ }_{p}^{2} / \Gamma$ where $b_{p}$ is the norm of the Burgers vector of the partial and $\Gamma$ is the SFE, as a function of the perfect dislocation' character.

Fig. 5: True stress-strain (normalised by $\mu$ the shear modulus)-curves of non-charged and hydrogen-charged nickel single crystals oriented for single slip. 
Figure 1:

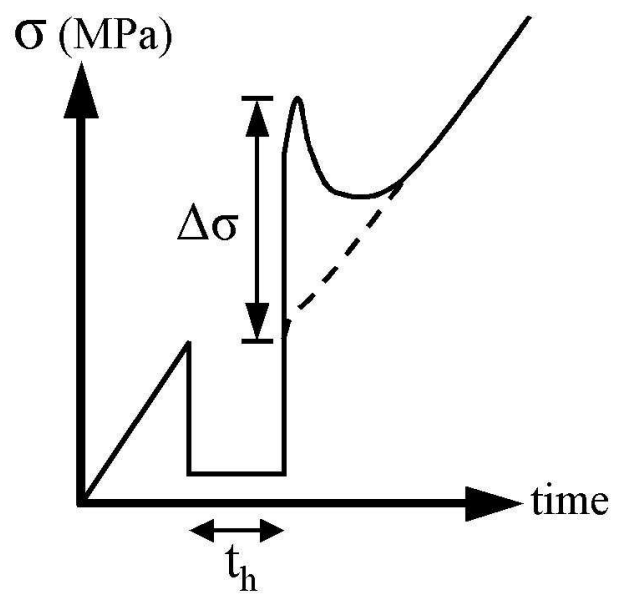


Figure 2:

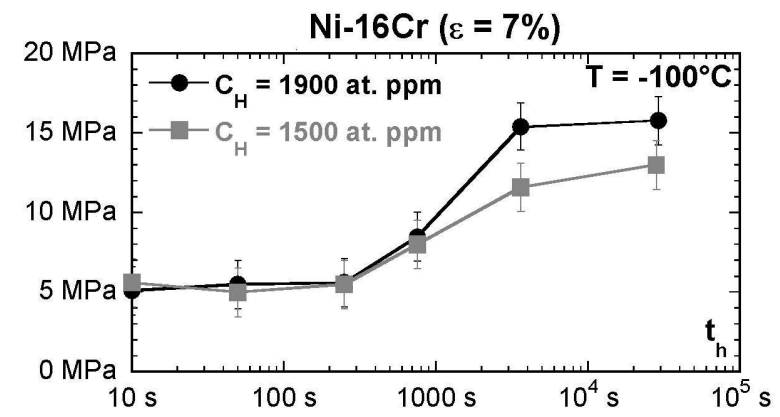

(a)

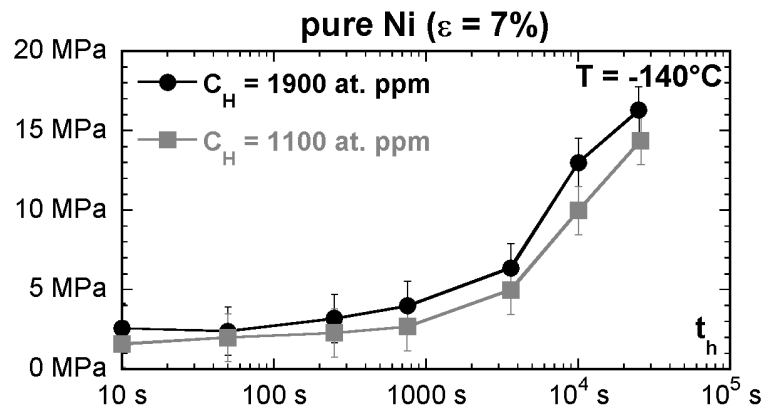

(b) 
Figure 3:

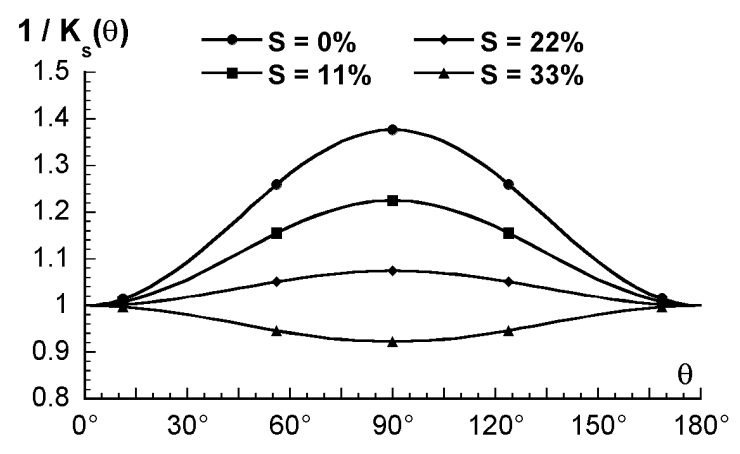

(a)

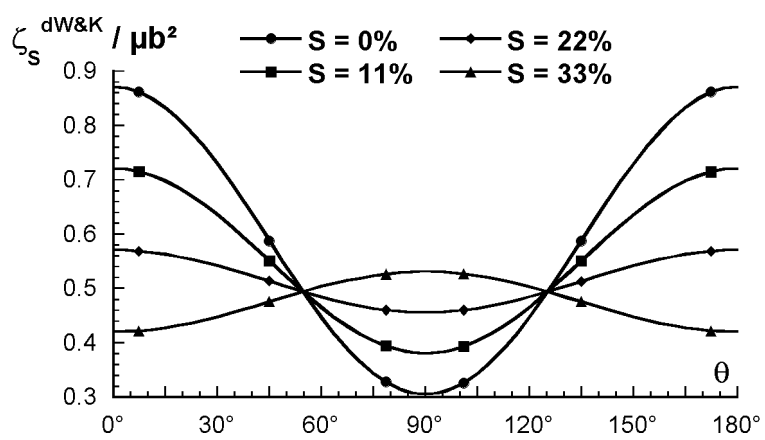

(b) 
Figure 4:

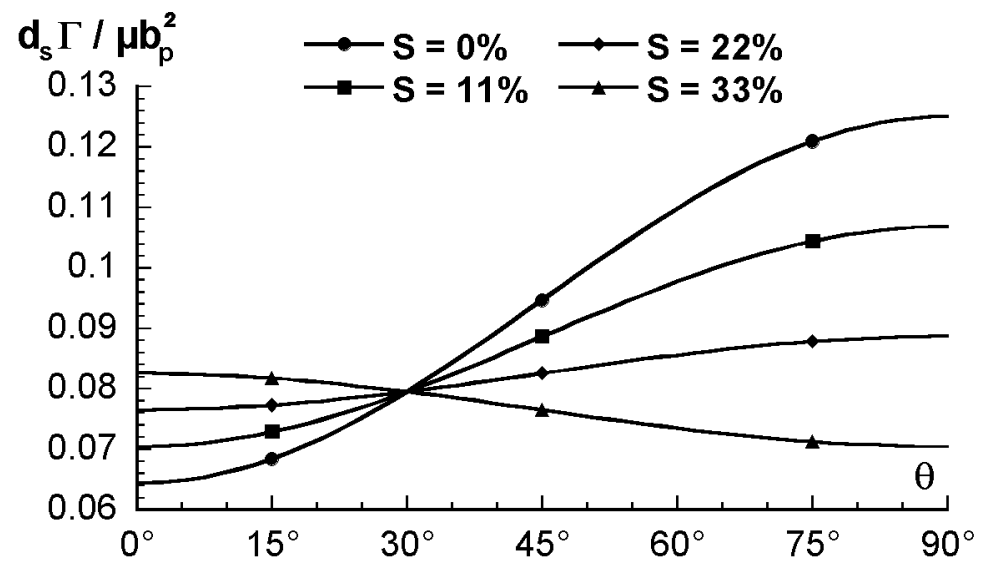


Figure 5:

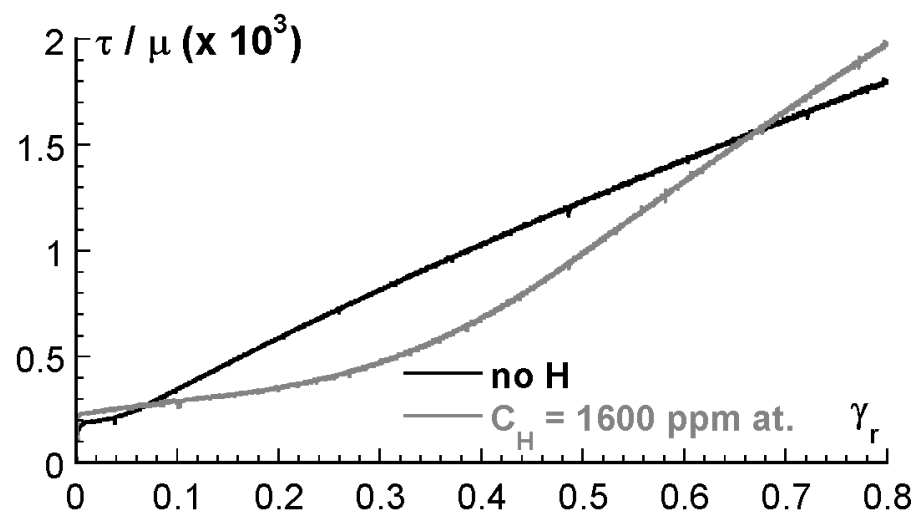

